\title{
Role of PCA in Offering Certifications and Other Related Services to POCUS Users Worldwide
}

\author{
Victor V Rao, MBBS, DMRD, RDMS (APCA) ${ }^{a}$, James A DellaValle, MD, MBA ${ }^{a}$, Samantha Forcum, M.Ed ${ }^{a}$, \\ Jasmine Rockett, Hannah Mason, MA, PMP ${ }^{a}$, Dale R. Cyr, MBA, CAE ${ }^{a}$ \\ ${ }^{a}$ Point of Care Certification Academy, Inteleos, Rockville, MD, USA \\ Received August 06, 2020; revision received August 09, 2020; accepted August 16, 2020

\begin{abstract}
The POCUS Certification Academy, a subdivision of Inteleos, a non-profit certification organization, is striving to promote global standards and international certifications in the field of POCUS to enhance clinician proficiency and ensure patient safety. By offering relevant rigorous assessments, developing continuing education requirements, and promoting the use of point-of-care ultrasound worldwide, the POCUS Certification Academy is laying the foundation for the highest global standards in POCUS credentialing.
\end{abstract}

Key words: Certification; Ultrasound education; POCUS; PCA; Inteleos; POCUS 25, MissionPOCUS, COVID-19.

Advanced Ultrasound in Diagnosis and Therapy 2020;03:260-263

DOI: $10.37015 /$ AUDT.2020.200058

\section{Introduction}

Point-of-care ultrasound (POCUS) has come a long way since its early use by the army during the Gulf war, where it was employed to quickly assess and triage wounded soldiers in the battlefield. After the war, many physicians introduced POCUS into their practice, especially in emergency medicine. In 2007, GE healthcare initiated a pilot project at two medical schools in the United States to introduce POCUS into the medical education curriculum. After seeing their successful integration, many other medical schools followed suit throughout the past decade. Simultaneously, innovative POCUS technology and equipment have continued to develop within the healthcare market. New pocketsized devices and cloud-based artificial intelligence (AI) units now range in price from approximately $\$ 2,000$ to $\$ 10,000$. The emergence of these low cost, appconnected, handheld POCUS devices will continue to further democratize the POCUS movement globally.

With an increase in the use and access to a variety of POCUS devices, the need for demonstrating proficiency in this clinical application has become critical to ensure patient safety. The POCUS Certification Academy (PCA) was created to do just that, by offering scientificallyvalidated certifications that are designed to confirm
POCUS-users' expertise and the delivery of high-quality healthcare. (Fig. 1). As a new member of the Inteleos' ARDMS and APCA family, the PCA is fostering an impressive membership of certified POCUS clinicians in over 100 countries across the globe (Fig. 2).

According to consumer data collected from the PCA, most POCUS practitioners are nontraditional users of ultrasound and have not had formal sonographic extended training in their scope of practice (Fig. 3). The traditional users of ultrasound diagnostic imaging are radiologists, cardiologists, and obstetrician/gynecologists among others. As the use of POCUS has become a part of nearly every practice setting, a huge number of medical professionals have begun enrolling in extensive training programs spanning over 1-3 years to be proficient in the use of handheld ultrasound. However, this is not always feasible for actively practicing physicians and healthcare workers, especially amidst a challenging training environment like the COVID-19 pandemic. Similarly, there is a lack of a defined, uniform curriculum for training efforts, which has led to some confusion amongst POCUS users. To remedy this, the POCUS Certification Academy created a comprehensive offering of POCUS certificates and educational bundles to address proper education and assessment of expertise in point-of-care ultrasound. 


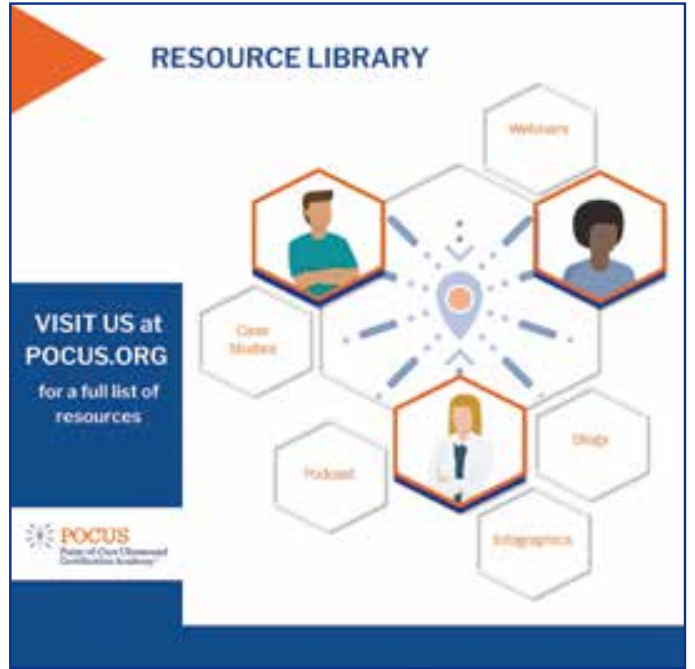

Figure 1 PCA POCUS Resource Library

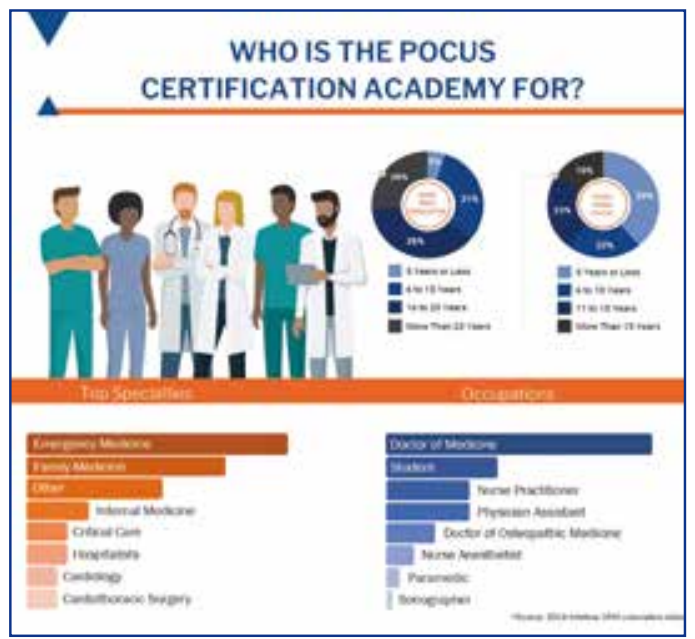

Figure 3 PCA Demographics user type

\section{Importance of POCUS Certification}

The purpose of independent validation in the healthcare industry is both to ensure patient safety, and to guarantee that healthcare workers who hold the credential have met a minimum standard for professional and ethical standards. Inteleos has a long-standing history of being independently accredited by the ISO 17024 standards for personnel certification. The PCA has been built to comply with these standards. According to a previous article published [1], initial certification required a considerable investment of time and money. Physicians seeking initial ABEM certification found both learning and professional benefits, with the most frequently reported being reinforcement of medical knowledge and more career opportunities. Another study article [2] concluded that maintaining certification was positively associated with physician performance scores on a set of HEDIS process measures.

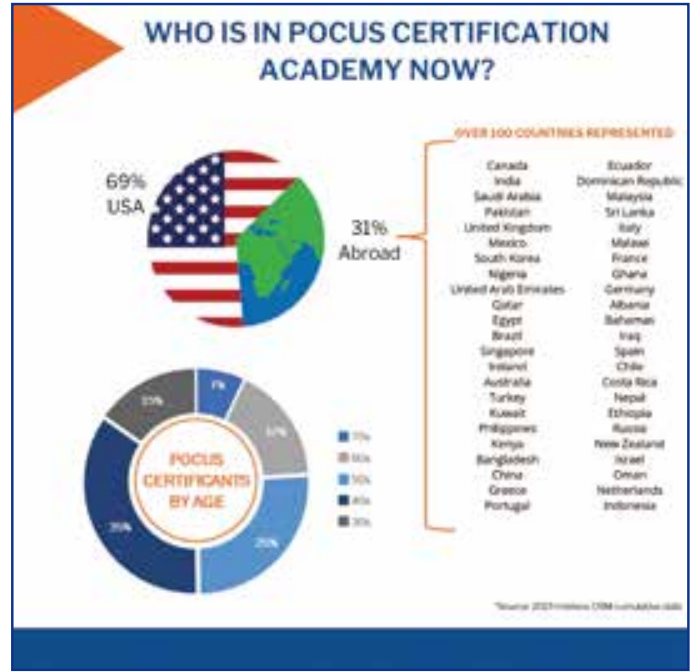

Figure 2 PCA Demographics Countries and Age

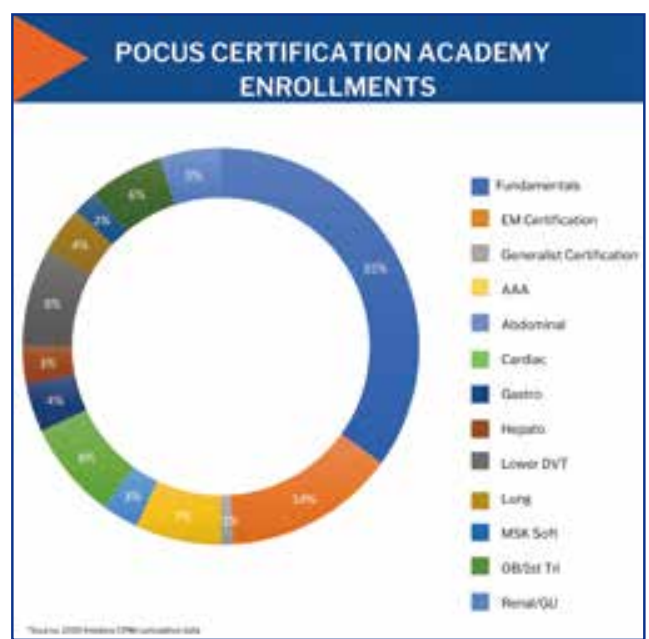

Figure 4 PCA enrollments by certificate type

\section{Current POCUS Certifications and Bundles}

Launched in 2017, the POCUS Certification Academy offers over a dozen certificates and certifications. As POCUS advances, more certifications and bundles will be offered (Fig. 4). The current individual certificates are as follows:

- POCUS Fundamentals Certificate

- Abdominal Aortic Aneurysm (AAA) Point-of-Care Ultrasound (POCUS) Certificate 1

- Abdominal Trauma Point-of-Care Ultrasound (POCUS) Certificate 1, 2 and 3

- Cardiac Point-of-Care Ultrasound (POCUS) Certificate

- Gastrointestinal Point-of-Care Ultrasound (POCUS) Certificate

- Hepatobiliary/Spleen Point-of-Care Ultrasound (POCUS) Certificate

- Lower Extremity Deep Vein Thrombosis (DVT) Point-of-Care Ultrasound (POCUS) Certificate 1 
- Lung Point-of-Care Ultrasound (POCUS) Certificate 1 and 2

- Musculoskeletal (MSK) Soft Tissue Point-of-Care Ultrasound (POCUS) Certificate

- Obstetrics/First Trimester Point-of-Care Ultrasound (POCUS) Certificate

- Renal/Genitourinary Point-of-Care Ultrasound (POCUS) Certificate

- 1 Includes components of the Rapid Ultrasound for Shock and Hypotension (RUSH) protocol.

- 2 Includes components of the Extended Focused Assessment with Sonography for Trauma (eFAST) protocol.

- 3 Includes components of the Focused Assessment with Sonography in Trauma (FAST)

The Certifications currently being offered are as follows:

- POCUS Generalist Certification

- POCUS Emergency Medicine Certification

\section{POCUS Certification Process}

Internationally accredited by ISO, Inteleos conducts psychometrically-validated test development activities which include: determining appropriate eligibility to sit for the exam, building an examination that accurately reflects the scope of practice, assuring the examinations are valid and reliable, and addressing continued competency for lifelong learning.

When building an ultrasound assessment, especially with the intention of offering it to a diverse set of practices and clinicians around the world, one of the most important steps is to partner with global subject matter experts (SMEs). Having a strong base of reliable, global subject matter experts is a critical component for building and maintaining a relevant exam for practice. POCUS practitioners themselves have the best understanding of their own scope of practice; incorporating insight and experience from the healthcare community is key in building appropriate credentialing exams.

\section{Eligibility and Application Requirements}

It is important that Inteleos establishes appropriate eligibility criteria that meets local or regional practitioner education and training environments. Since there is not currently a global set of standards for POCUS practice, it is important that Inteleos analyze practice patterns, conduct market research, and utilize input from SMEs and local regulatory groups to determine appropriate eligibility requirements. Eligibility requirements primarily include educational parameters, in addition to documented clinical experience.
POCUS and its Critical Role in the COVID-19

\section{Pandemic}

Amidst the current COVID-19 pandemic, the use of POCUS has taken center stage especially in treating ARDS. Commonly, referred to as "the stethoscope of the 21 st century", point-of-care ultrasound has proven to be an essential tool in emergency departments, ICUs, and general hospital practice in managing patients during this pandemic. POCUS is primarily being used to monitor and triage lung, and cardiac and DVT complications associated with the novel coronavirus.

\section{The POCUS 25}

From its onset, there has been lack of clarity about a unified POCUS scope of practice. POCUS, in its original intent, was designed to be an extension of the physical exam, used to answer several yes/no questions and to typically be completed in a matter of minutes. It was envisioned to fit into the busy schedule of a clinician who can use modern diagnostic, imaging tools to enhance and or to confirm physical exam findings, detect normal or abnormal anatomy and pathophysiological changes, and to confirm and or eliminate a possible differential diagnosis. POCUS can even be used to assist a healthcare provider in effectively performing an ultrasound-guided procedure by providing visual support of structures inside the body and the needle tip as it traverses through the tissues.

With the growing adoption of POCUS across a diversity of practice settings, so too comes a need to better define the appropriate curriculum for POCUS education and assessment. In 2019, the POCUS Certification Academy launched POCUS25-a participatory longitudinal research study designed to collect community feedback on 25 core practice domains in point-of-care ultrasound. Modeled after the Stanford 25 , the POCUS25 is a psychometrically validated list of POCUS practice domains that will act as a guide to determine the priorities for POCUS education and certification. The skills list is not intended to limit a POCUS user, and the PCA encourages clinicians to learn skills beyond those listed. The initial 25 practice domains included in the study are intended to lead the future design of POCUS teaching, training, and credentialing that can translate into clinical practice in various geographic locations.

With responses from over 50 countries thus far, the PCA aims to achieve global participation from POCUS users in all 195 countries and territories. After reaching the threshold of responses needed, the POCUS25 study will be published for the global healthcare community to access and utilize as a reference guide for informed 
POCUS integration into all practice settings.

\section{MissionPOCUS}

In addition to research, the POCUS Certification Academy is proud to support the integration of POCUS practice into new rural and under-resourced locations. Through their annual MissionPOCUS initiative, the PCA has offered training, certification, and handheld ultrasound devices to recipients of past projects, most recently clinics in Haiti and Kenya. In their recent Summer announcement, the PCA selected Médecins Sans Frontières (MSF), also known as Doctors without Borders, as the MissionPOCUS project for the 2020 fiscal year. The PCA will award MSF a grant that will establish a pilot program between the two organizations to evaluate the possibility of integrating POCUS certification into MSF's POCUS education and training processes. The pilot is focused on developing a unique POCUS certification that will translate across resource-limited areas, with the goal of expanding the proficient use of point-of-care ultrasound to clinicians around the globe.

\section{Invitation to Participate}

Whether you are new to using point-of-care ultrasound or have been practicing ultrasound for years, the POCUS Certification Academy invites you to join the growing global POCUS community.

Have you taken the POCUS25 survey yet? Make sure your country and region are represented by taking the survey today at POCUS.org or by scanning the QR code below!

Interested in volunteering or having your organization as the next MissionPOCUS project for the 2021? Complete the application on POCUS.org or by scanning the $\mathrm{QR}$ code below!

\section{QR Codes for Quick Access to Website}

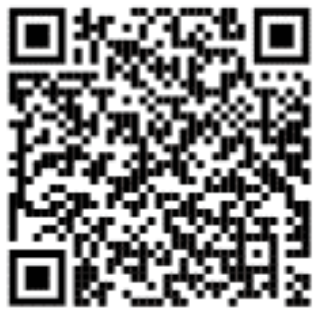

Get Certified!

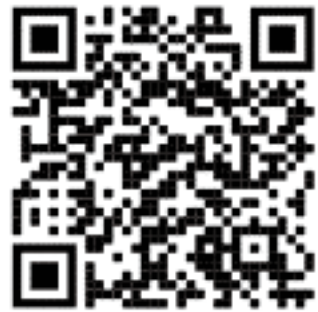

Take the POCUS25 Survey!

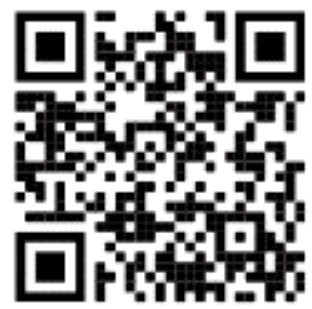

Volunteer with MissionPOCUS!

\section{Conflict of Interest}

The authors are employees of Inteleos: Victor V Rao, MBBS, DMRD, RDMS (APCA), Manager of Global Clinical Content; James A DellaValle, MD, MBA, Chairman of Inteleos' POCUS Council, Samantha Forcum, M.Ed, Global Manager of Outreach; Jasmine Rockett, Deputy Director of POCUS Certification Academy, Hannah Mason, MA, PMP, Manager of Business Development, Inteleos, and Dale R. Cyr, MBA, CAE, Executive Director and CEO, Inteleos.

\section{References}

[1] Baren JM, Beeson MS, Chudnofsky CR, Goyal DG, Kowalenko $\mathrm{T}$, Mallory MNS, et al. Costs and benefits of initial certification for emergency medicine residency graduates. J Grad Med Educ 2019; 11: 649-653.

[2] Gray B, Vandergrift J, Landon B, Reschovsky J, Lipner R. Associations between American board of internal medicine maintenance of certification status and performance on a set of healthcare effectiveness data and information set (HEDIS) process measures. Ann Intern Med 2018; 169: 97-105.

[3] American College of Emergency Physicians. Ultrasound guidelines: emergency, point-of-care and clinical ultrasound guidelines in medicine. Ann Emerg Med 2017; 69: e27-e54.

[4] Frankel HL, Kirkpatrick AW, Elbarbary M, Blaivas M, Desai H, Evans D, et al. Guidelines for the appropriate use of bedside general and cardiac ultrasonography in the evaluation of critically ill patients- part i: general ultrasonography. Crit Care Med 2015; 43: 2479-2502.

[5] Hoppmann RA, Rao VV, Bell F, Poston MB, Howe DB, Riffle S, et al. The evolution of an integrated ultrasound curriculum (iUSC) for medical students: 9-year experience. Crit Ultrasound J 2015; 7: 18.

[6] Expert Round Table on Echocardiography in ICU. International consensus statement on training standards for advanced critical care echocardiography. Intensive Care Med 2014; 40: 654-666.

[7] Levitov A, Frankel HL, Blaivas M, Kirkpatrick AW, Su E, Evans $\mathrm{D}$, et al. Guidelines for the appropriate use of bedside general and cardiac ultrasonography in the evaluation of critically ill patients-part ii: cardiac ultrasonography. Crit Care Med 2016; 44: 1206-1227.

[8] Lewis D, Rang L, Kim D, Robichaud L, Kwan C, Pham C, et al. Recommendations for the use of point-of-care ultrasound (POCUS) by emergency physicians in Canada. CJEM 2019; 21: 721-726.

[9] Mayo PH, Beaulieu Y, Doelken P, Feller-Kopman D, Harrod C, Kaplan A, et al. American College of Chest Physicians/La Société de Réanimation de Langue Française statement on competence in critical care ultrasonography. Chest 2009; 135: 1050-1060.

[10] Schnobrich DJ, Gladding S, Olson AP, Duran-Nelson A. Point-ofcare ultrasound in internal medicine: a national survey of educational leadership. J Grad Med Educ 2013; 5: 498-502.

[11] Via G, Hussain A, Wells M, Reardon R, ElBarbary M, Noble VE, et al. International evidence-based recommendations for focused cardiac ultrasound. J Am Soc Echocardiogr 2014; 27: 683.e1-683.e33.

[12] Volpicelli G, Elbarbary M, Blaivas M, Lichtenstein DA, Mathis G, Kirkpatrick AW, et al. International evidence-based recommendations for point-of-care lung ultrasound. Intensive Care Med 2012; 38: 577 591. 